\title{
Ampliação do Nível de Sustentabilidade Ambiental de CalÇados Ecológicos a Partir da Avaliação do CiClo de Vida
}

Aline Medeiros Dalbem (alinemedeirosdalbem@gmail.com) - Globo Comunicação e Participações S.A., Rappi.

Fernando Henrique Lermen (fernando.lermen@unespar.edu.br) - Universidade Estadual do Paraná, UNESPAR.

Ângela de Moura Ferreira Danilevicz (angelamfd@producao.ufrgs.br) - Universidade Federal do Rio Grande do Sul, UFRGS.

\section{RESUMO}

Com base na tendência mundial de preocupação com o meio ambiente e no modelo tradicional de consumo caracterizado pela renovação constante de peças e pelo incentivo ao descarte, passam a existir esforços para aproximar o conceito de desenvolvimento sustentável ao da moda. O presente trabalho tem por objetivo identificar oportunidades de ampliação do nível de sustentabilidade ambiental, bem como divulgar o real impacto ambiental da produção. Para tanto foi explicitado o passo-a-passo para o planejamento e desenvolvimento da avaliação do ciclo de vida para o setor calçadista, com foco na mensuração dos impactos ambientais. Os procedimentos metodológicos foram estruturados conforme as etapas da ACV: definição de objetivos e escopo; análise de inventário; avaliação dos impactos ambientais e interpretação dos resultados. A fim de avaliar os potenciais impactos sugere-se aplicação das metodologias CML, CED, Eco-indicator 99 e o cálculo de pegada de carbono. Os resultados sugerem que o transporte das matérias primas e do produto contribuíram significativamente no resultado. Conclui-se que o ciclo em malha fechada e uma economia circular elevam o nível de sustentabilidade do produto. Por fim, sugere-se a aplicação de matérias biodegradáveis e o uso de recursos renováveis a fim de ampliar o nível de sustentabilidade do produto.

Palavras-chave: Avaliação do Ciclo de Vida; Sustentabilidade Ambiental; Pegada de Carbono; Inovação Sustentável; Indústria Calçadista. 


\section{INTRODUÇÃO}

A moda compreende um universo que engloba roupas, calçados, dentre outros artigos; ela reflete as ansiedades do mundo moderno e é um fenômeno altamente dinâmico que busca na sua essência representar os desejos momentâneos das pessoas (TODESCHINI et al., 2020; BRYDGES et al., 2021). Para refletir tendências e atender prontamente às necessidades dos consumidores, a moda foi se moldando com o tempo. Assim, a indústria também passou por transformações, as produções passaram a ser menores e mais rápidas uma vez que o número de coleções e temporadas aumentou, encurtando o tempo de espera dos consumidores (JUNG; JIN, 2016; DJAFAROVA; BOWES, 2021).

Segundo Shen (2014) quando uma empresa do mundo fashion busca aderir à consciência ambiental como um todo, um elo importante é desenvolver uma cadeia de suprimentos sustentável. Dessa forma, todas as etapas do processo produtivo desde da origem da matériaprima até o descarte pelo consumidor 'consciente' são significativas e os impactos ambientais de cada fase devem ser considerados.

Manzini e Vezzoli (2002) definem design para a sustentabilidade ambiental como uma estratégia de projetar produtos que resultem em alta qualidade social com o mínimo desperdício e prejuízo para a natureza; e que, no futuro, produzam impactos positivos na sociedade e no meio ambiente. Essa estratégia é também uma forma de pressionar as indústrias, seja de moda ou de outros produtos, para diminuírem o prejuízo que causam ao meio ambiente. Hasan et al. (2019) ressaltam ainda que negócios verdes podem, além de melhorar o meio ambiente, aumentar a relação custo-benefício do produto.

Nesse contexto, o mapeamento do ciclo de vida do produto é essencial para o desenvolvimento sustentável. Dentro da cadeia de suprimentos, faz-se necessário o fortalecimento do vínculo com fornecedores e parceiros, o que pode resultar tanto em eficácia quanto eficiência produtiva, bem como em uma cadeia verde mais consolidada.

O objetivo desse trabalho é realizar o planejamento e desenvolvimento da avaliação do ciclo de vida de um par de calçado ecológico a fim de identificar oportunidades de ampliação do nível de sustentabilidade ambiental e de divulgar o real impacto ambiental da produção. Com isso, espera-se o aperfeiçoamento do produto, alinhando-o cada vez mais aos valores de brasilidade, valorização de materiais ecológicos e veganismo da empresa analisada, bem como o fortalecimento de sua marca. De maneira a delimitar o presente estudo, salienta-se que os aspectos econômicos e sociais relacionados à implantação de potenciais mudanças 
estão excluídos da análise, assim como os riscos e o trabalho humano associados. Além disso, também não é foco a verificação de viabilidade mercadológica do produto avaliado.

\section{REVISÃO TEÓRICA}

Inicialmente, moda e sustentabilidade ambiental parecem conceitos contraditórios. Por um lado, a moda estimula um grande número de coleções e um curto ciclo de vida dos produtos, enquanto, por outro lado, a sustentabilidade ambiental preconiza o equilíbrio entre o meio ambiente e a produção, com foco na reutilização e reuso (OZDAMAR ERTEKIN; ATIK, 2015).

Nesse contexto, a indústria calçadista se depara com o desafio de planejar a reciclagem do produto ao final do seu ciclo de vida, uma vez que a separação dos componentes se torna complexa, considerando que aproximadamente 40 materiais diferentes podem ser usados na fabricação de um sapato. Quando este planejamento não ocorre, gera-se um problema ambiental significativo, por meio de calçados que, frequentemente, são descartados em aterros sanitários (STAIKOS et al., 2006).

Dessa forma, uma visão sistemática de todas as etapas se torna fundamental para avaliar impacto ambiental dos produtos. Nesse contexto, Hendrickson et al. (1998) apontam a avaliação do ciclo de vida como uma poderosa ferramenta para analisar o nível de sustentabilidade do produto e detectar oportunidades de melhoria.

A abordagem da avaliação do ciclo de vida (ACV) é amplamente utilizada como ferramenta para tomada de decisão em questões referentes a gestão ambiental (JOSHI; COLLEGE, 2000). A ACV apresenta uma característica única frente a outras ferramentas de estudo do nível de sustentabilidade ambiental de produtos, pois considera cada etapa no ciclo de vida do produto. Assim, o escopo abrange desde a avaliação da extração da matéria-prima até o descarte final. Nesse contexto, a avaliação do ciclo de vida é ainda mais vantajosa para o estudo do nível de sustentabilidade ambiental no setor calçadista, uma vez que o maior desafio atual do setor é o descarte em aterros.

\section{METODOLOGIA}

O desenvolvimento deste trabalho utilizou como base as quatro etapas da avaliação do ciclo de vida de produto, ilustradas na Figura 1 e descritas a seguir. 


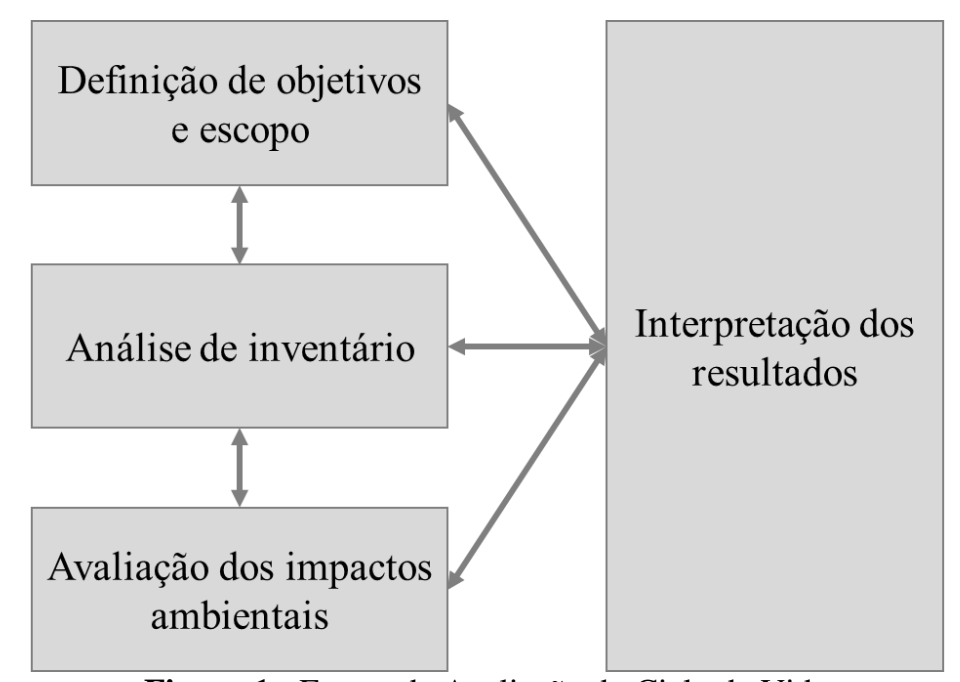

Figura 1 - Etapas da Avaliação do Ciclo de Vida

Fonte: NBR ISO 14.040 (ABNT, 2001).

Na primeira etapa, o objetivo e o escopo da pesquisa foram definidos, delimitando-se os seguintes itens: unidade funcional, fronteiras do sistema, suposições, limitações, procedimentos e tipo de análise crítica. O escopo da avaliação foi estabelecido com base nas limitações de informações dos fornecedores locais e relevância no estudo do nível de sustentabilidade do produto. A avaliação exclui aspectos econômicos, sociais, assim como riscos e viabilidade mercadológica do calçado ecológico. Nessa etapa também foi desenvolvido o sistema de produto da ACV.

Na segunda etapa, a análise do inventário, foi realizado um levantamento de dados sobre os processos, matérias primas e transporte dos fornecedores até a empresa. Na terceira etapa, foi realizada a avaliação qualitativa dos principais contribuintes de impactos ambientais. $\mathrm{Na}$ última e quarta etapa, os resultados encontrados anteriormente foram interpretados. As respostas obtidas foram associadas a fim de detalhar o nível de sustentabilidade do calçado ecológico.

\section{RESULTADOS E DISCUSSÃO}

Nesta seção são apresentados os resultados e discussões da pesquisa, assim como sugestões de trabalhos futuros.

\subsection{Definição de objetivos e escopo}

O objetivo da avaliação do ciclo de vida do produto é estimar o valor de pegada de carbono de um par de calçado a fim identificar oportunidades de ampliação do nível de sustentabilidade ambiental e de divulgar o real impacto ambiental da produção em Porto Alegre (2019). A 
partir da escolha do produto no portfólio da empresa, representado na Figura 2, o escopo da ACV foi delimitado.

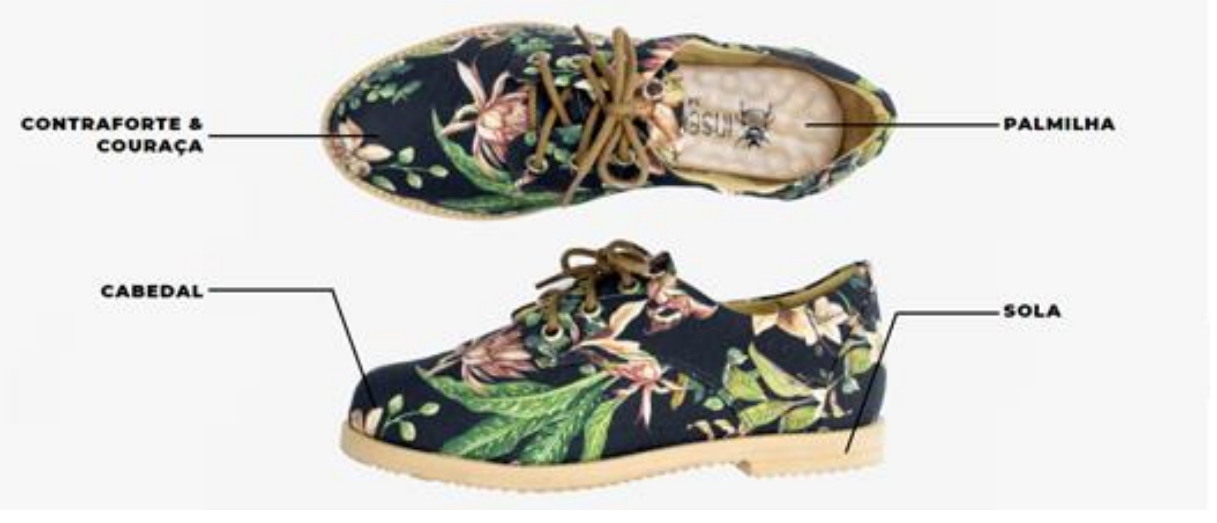

Figura 2 - Anatomia simplificada do calçado

Fonte: Insecta Shoes (2019).

O sistema de produto inclui a produção das matérias primas, manufatura do calçado, venda e logística reversa pós consumo do produto. Nesse contexto, a unidade funcional do sistema foi definida como 1 par de calçado. O sistema de produto da ACV foi desenhado, conforme Figura 3.

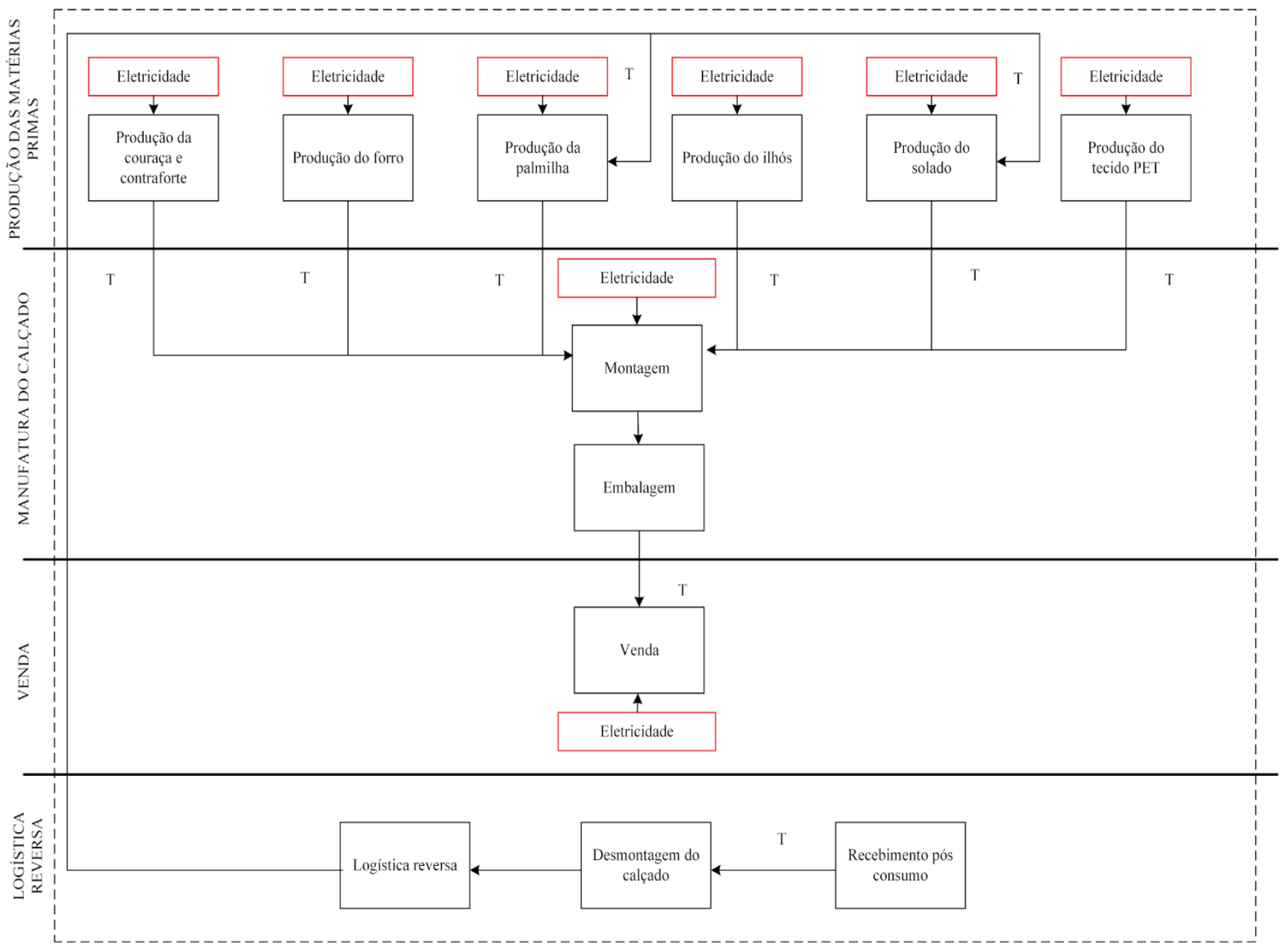


Figura 3 - Sistema de produto da ACV.

Fonte: Elaborado pelos autores.

O sistema de produto é dividido em quatro fases: produção das matérias primas, manufatura do calçado, venda e logística reversa. Na segunda parte, a manufatura do calçado é iniciada pela montagem das peças. A seguir os calçados são encaixotados e as embalagens são preparadas para envio até o centro de distribuição em São Paulo. Na terceira etapa, a fase da venda inclui os processos internos e a energia necessário na operação da empresa. Nessa etapa são consideradas operações de lojas físicas, assim como logística de e-commerce, marketing, procedimentos administrativos e outras atividades de manutenção da empresa. Por fim, a logística reversa inclui o recebimento pós consumo do calçado, desmontagem do sapato no ateliê e retorno dos insumos no ciclo de produção das palmilhas e do solado.

\subsection{Análise de inventário}

Com base do sistema de produto, foi realizada a coleta de dados para análise de inventário. Primeiramente foram estimadas as distâncias entre fornecedores a partir de rotas reais, assim como o peso em kg de cada insumo para compor a tabela de dados de transporte de cada fase. De posse dos dados de transporte, foi realizado o inventário total do ciclo do calçado considerando as matérias primas e embalagem, assim como a energia necessária em cada etapa, conforme Tabela 1.

Tabela 1 - Dados para análise de inventário.

\begin{tabular}{ccccc}
\hline \multirow{2}{*}{ Fase } & Tipo & Artigo & Quantidade em 1 par de calçado \\
\cline { 3 - 5 } & Entrada & Valor & Unidade \\
& Entrada & Atacadores & 0,2000 & metro linear \\
unidade
\end{tabular}


Com base na Tabela 1, foi possível verificar a demanda de energia de cada fase. Notou-se que a produção de palmilha e de solado, as quais são responsáveis pelo fechamento de ciclo do calçado, pois nesses processos são reinseridos o calçado desmontado pós o consumo, apresentam valores elevados de energia por par de sapato. Além disso, foi possível constatar a dimensão da etapa de manufatura do calçado e de venda, sendo que a montagem representa mais da metade da energia total necessário no ciclo.

Apesar da elevada demanda de energia na fase de manufatura e de venda do calçado, a magnitude dos valores de transporte se destaca no processo, visto que a maioria das matérias primas e montagem são localizadas no estado do Rio Grande do Sul, enquanto o centro de distribuição e maior público de compra está localizado no estado de São Paulo.

\subsection{Avaliação qualitativa dos impactos ambientais}

A partir da análise de inventário foi possível avaliar os potenciais impactos ambientais associados ao ciclo de um par de calçado. A fim de avaliar a pegada de carbono foi calculado um valor preliminar a partir do fator de emissão de $\mathrm{CO}_{2}$ pela geração de energia elétrica no Sistema Interligado Nacional do Brasil médio mensal de 2019 de $0,47 \quad \mathrm{kgCO}_{2} / \mathrm{kWh}$. Considerando que a soma das energias de todas as etapas desenhadas no sistema de produto foi de 17,5 MJ, aproximadamente 4,9 $\mathrm{kWh}$, a parcela da energia correspondeu a 2,3 $\mathrm{kg}$ de $\mathrm{CO}_{2}$

Além da energia, foi necessário considerar os dados de transporte no cálculo de pegada de carbono, visto que o ciclo completo do calçado inclui grandes distâncias. Considerando dados do Inventário Nacional de Emissões Atmosféricas por Veículos Automotores Rodoviários (MMA 2014) veículos movidos a diesel apresentam um fator de emissão de $\mathrm{CO}_{2}$ de aproximadamente 2,6 kg/L $/ \mathrm{L}_{\text {diesel }}$; sendo que os consumos são em média $9,1 \mathrm{~km} / \mathrm{L}_{\text {diesel }}$ para um caminhão semileve e $3,4 \mathrm{~km} / \mathrm{L}_{\text {diesel }}$ para um caminhão semipesado. De posse dos dados de transporte, foi possível estimar a emissão de $1834,1 \mathrm{~kg}$ de $\mathrm{CO}_{2}$ devido ao transporte.

Assim, foi previsto o valor de $1836,4 \mathrm{~kg}$ de $\mathrm{CO}_{2}$ por par de calçado para um futuro cálculo de pegada de carbono com aplicação da ACV. Sendo que mais de $99 \%$ desse total é proveniente do transporte rodoviário do ciclo do calçado. Desse modo, notou-se a importância da compensação ou neutralização de carbono como medida remediadora a fim de mitigar o impacto ambiental do aquecimento global.

\subsection{Interpretação dos resultados}


Com base no desenho do sistema de produto, conclui-se que, apesar da manufatura do calçado envolver elementos de diferentes fontes com tecido e borracha, o ciclo de vida do produto é relativamente simples. Uma vez que os insumos pós consumo do calçado voltam para o ciclo na etapa de produção da matéria prima e os fornecedores possuem processos internos de reciclagem e reuso dos recursos, formando, assim, a malha fechada do sistema de produto. Winkler (2011) confirma que muitos impactos ambientais negativos, como resíduos, elevado consumo de energia, processos de transporte e embalagem são evitados em sistemas de malha fechada.

Nesse cenário, uma economia circular melhora a sustentabilidade e pode beneficiar o desempenho econômico e ambiental da empresa. No cenário atual no qual os padrões de consumo contribuem a um aumento na geração de lixos é imprescindível o gerenciamento eficaz de resíduos. Nesse contexto, considerando a relativa curta vida útil do calçado, a malha fechada é essencial para reduzir os impactos ambientais do calçado, uma vez que é crescente o volume de sapatos descartados em aterros sanitários (STAIKOS; RAHIMIFARD, 2007).

Nesse sentido, o processo de reciclagem é essencial e a utilização dos materiais provenientes desse recurso apresentam impacto ambiental reduzido frente a materiais virgens como borracha na produção do solado. Staikos e Rahimifard (2007) afirmam que os riscos mais graves para o meio ambiente são encontrados com fornecedores de produtos semiacabados e componentes, como o couro que é produzido por curtimento no qual o cromo, agente do processo, é altamente tóxico, contribui para formação de ozônio ao nível do solo e pode ser cancerígeno.

A fim de avaliar quantitativamente os impactos ambientais, a literatura indica o cálculo de pegada de carbono como indicador amplamente utilizado. No entanto, apesar do seu uso universal, há uma aparente falta de definições acadêmicas sobre como calcular a emissão de $\mathrm{CO}_{2}$ (WIEDMANN; MINX, 2007). Assim, a falta de padrão no cálculo não permite a comparação entre valores de pegada de carbono entre diferentes empresas.

\section{CONCLUSÃO}

À medida que a preocupação com o meio ambiente cresce, o consumo consciente se torna tendência de comportamento. Nesse cenário, os consumidores são os agentes de mudança uma vez que questionam e exigem das indústrias, marcas e lojas um posicionamento frente a questão ambiental. O presente trabalho buscou avaliar o nível de sustentabilidade do calçado modelo Oxford da linha de estamparia própria da empresa Insecta Shoes, em Porto Alegre, 
com ênfase na identificação de oportunidades de ampliação desse nível. Para isso, foi realizado o planejamento e desenvolvimento de ACV para o setor calçadista, com foco na mensuração dos impactos ambientais.

Dentre as limitações do presente trabalho, podem ser citadas: (i) a confiabilidade da coleta de dados junto aos fornecedores; (ii) a incerteza em relação às rotas de transporte e aos tipos veículos utilizados no processo; e (iii) a falta de banco de dados brasileiros na aplicação de ACV. A partir da análise do inventário, foi possível concluir sobre a importância do desenvolvimento de uma logística local, considerando a magnitude dos impactos gerados pelo transporte. Além disso, o sistema em ciclo fechamento e a economia circular se destacam na ampliação do nível de sustentabilidade do produto. Também foi possível inferir que é essencial um vínculo de confiança, em uma lógica ganha-ganha, com fornecedores e parceiros, de modo a garantir o cuidado com meio ambiente e consolidar uma cadeia de suprimentos verde.

Não obstante, notou-se que o ciclo de um produto, mesmo sendo ecológico, apresenta um valor de pegada de carbono elevado, o que ressalta a importância da compensação de carbono para qualquer processo produtivo. Por fim, notou-se o desenvolvimento sustentável agrega valor ao produto, melhorando a relação de consumo e a reputação da marca. Assim, como sugestões de pesquisas futuras é possível indicar, primeiramente, a aplicação desta sequência metodológica em outros tipos de calçados, de maneira a definir um ranking de pegada de carbono associada aos produtos da empresa. A partir desse ranking é possível subsidiar o processo decisório de sustentabilidade em relação à inovação, melhoria e descontinuidade de produtos.

Além disso, sugere-se a aplicação dessa sequência metodológica em diferentes tipos de produtos, de maneira a disseminar a ACV, ampliar a cultura pela sustentabilidade, bem como para contribuir para a definição de dados brasileiros para a aplicação de ACV. Considerando a magnitude elevada do impacto ambiental do descarte de calçado, sugere-se também o estudo da aplicação de materiais biodegradáveis, juntamente com o uso de recursos renováveis, como solução em médio em longo prazo para a manufatura de calçados. Por fim, a pesquisa e a avaliação de modelos de negócio facilitadores da reutilização dos produtos são incentivadas.

\section{REFERÊNCIAS}

ASSOCIAÇÃO BRASILEIRA DE NORMAS TÉCNICAS - ABNT. ABNT NBR ISO 14040 Gestão ambiental - Avaliação do ciclo de vida - Princípios e estrutura. Associação Brasileira de Normas Técnicas, p. 1-22, 2001. 
BRYDGES, Taylor. Closing the loop on take, make, waste: Investigating circular economy practices in the Swedish fashion industry. Journal of Cleaner Production, v. 293, p. 126245, 2021.

CACHON, G. P.; SWINNEY, R. The Value of Fast Fashion: Quick Response, Enhanced Design, and Strategic Consumer Behavior. Management Science, v. 57, n. 4, p. 778-795, 2011.

CATALDI, C.; DICKSON, M.; GROVER, C. Slow Fashion: Tailoring a strategic industry approach towards sustainability. Sustainability in fashion and textiles: VALUES, DESIGN, PROCUTION AND CONSUMPTION, p. 22-46, 2013.

COLTRO, L. et al. Avaliação do ciclo de vida como instrumento de gestão. Campinas: Cetea/Ital, v. 75, 2007.

CRAIGHILL, A. L.; POWELL, J. C. Lifecycle assessment and economic evaluation of recycling: A case study. Resources, Conservation and Recycling, v. 17, n. 2, p. 75-96, 1 ago. 1996.

CRESPO MENDES, N.; BUENO, C.; OMETTO, A. R. Avaliação de Impacto do Ciclo de Vida: revisão dos principais métodos Palavras-chave. Production, v. 26, n. 1, p. 160-175, 2016.

DE BRITO, M. P.; CARBONE, V.; BLANQUART, C. M. Towards a sustainable fashion retail supply chain in Europe: Organisation and performance. International Journal of Production Economics, v. 114, n. 2, p. 534-553, 2008.

EKVALL, T. et al. Recent developments in Life Cycle Assessment. Journal of Environmental Management, v. 91, n. 1, p. 1-21, 2009.

ELKINGTON, J. Towards the Sustainable Corporation: Win-Win-Win Business Strategies for Sustainable Development. California Management Review, v. 36, n. 2, p. 90-100, 1 jan. 1994.

HANDBOOK, I. L. C. D. Analysis of existing Environmental Impact Assessment methodologies for use in Life Cycle Assessment. Joint Research Centre-European commission, 2010.

FLETCHER, K. Slow Fashion: An Invitation for Systems Change. n. November 2010, 2016.

FOUNDATION, E. M. Economia Circular: Disponível em: <https://www.ellenmacarthurfoundation.org/pt/economia-circular-1/conceito>. Acesso em: 20 jun. 2019.

FOX, N.; WARD, K. Health, Ethics and Environment: a Qualitative Study of Vegetarian Motivations. Disponível em: <http://eprints.whiterose.ac.uk/3741/1/foxn1.pdf>. Acesso em: 20 jun. 2019.

FRISCHKNECHT, R. et al. Implementation of Life Cycle Impact Assessment Methods. American Midland Naturalist, v. 150, n. 3, p. 1-151, 2007.

From Nike Reuse-A-Shoe to Nike Grind - Our ldb. Disponível em: <https://www.ourldb.com/news/2018/1/21/from-nike-reuse-a-shoe-to-nike-grind>. Acesso em: 24 abr. 2019.

GIL, A. C. Como elaborar projetos de pesquisa. São Paulo, v. 5, n. 61, p. 16-17, 2002.

GOEDKOOP, M.; SPRIENSMA, R. The Eco-indicator 99. A damage-oriented method for Life Cycle Impact Assessment Methodology Report. Pre-Consultants, The Netherlands. https://teclim.ufba.br/jsf/indicadores/holan\%20ecoindicator\%2099.pdf. 2000.

GUEDES, M. DA G. Ethical fashion brands: promotion approach or a real value. International Conference Fashion and Communication, p. 1-15, 2011.

GUINEE, J. B. Handbook on life cycle assessment operational guide to the ISO standards. The International Journal of Life Cycle Assessment, v. 7, n. 5, p. 311-313, set. 2002.

HART, S. L.; MILSTEIN, M. B. Creating sustainable value. Academy of Management Executive, v. 17, n. 2, p. 56-67, 2011. 
HASAN, M. M. et al. Green business value chain: a systematic review. Sustainable Production and Consumption, v. 20, p. 326-339, 1 out. 2019.

HAZARIKA, N.; ZHANG, X. Evolving theories of eco-innovation: A systematic review. Sustainable Production and Consumption, v. 19, p. 64-78, 1 jul. 2019.

HENDRICKSON, C. T. et al. Economic Input-Output Models for Environmental Life-Cycle Assessment. Environmental Science \& Technology, v. 32, n. 7, p. 184a-191a, 1998.

HERVA, M.; ÁlVAREZ, A.; ROCA, E. Sustainable and safe design of footwear integrating ecological footprint and risk criteria. Journal of Hazardous Materials, v. 192, n. 3, p. 1876-1881, 2011.

JOHANSSON, E. Slow Fashion - the answer to a sustainable fashion industry. p. 1-94, 2010.

JOSHI, S.; COLLEGE, J. M. Product Environmental Life-Cycle Assessment Using Input-Output Techniques. Journal of Industrial Ecology, v. 3, n. 2, p. 95-120, 2000.

JUCÁ, J. F. T., BASTOS, E. G., MELO, V. L. A. Monitoramento ambiental do aterro de resíduos sólidos da Muribeca - PE. 19 o Congresso Brasileiro de Engenharia Sanitária e Ambiental, ABES Congresso Brasileiro de Engenharia Sanitária e Ambiental, ABES, n. 1, p. 037, 1997.

JUNG, S.; JIN, B. Sustainable Development of Slow Fashion Businesses : Customer Value Approach. 2016.

KILIÇ, E. et al. Corporate carbon footprint for country Climate Change mitigation: A case study of a tannery in Turkey. Science of the Total Environment, v. 635, p. 60-69, 2018.

KUNAMANENI, S.; JASSI, S.; HOANG, D. Promoting reuse behaviour: Challenges and strategies for repeat purchase, low-involvement products. Sustainable Production and Consumption, v. 20, p. 253-272, 1 out. 2019.

SVENDSEN, L. Moda: uma filosofia. Zahar, 2010.

LOPES, N. K. S. E L. SUSTENTABILIDADE AMBIENTAL: UM DESAFIO PARA A MODA. Modapalavra e-periódico, p. 31-42, 2018.

LOPES SILVA, D. A. et al. Why using different Life Cycle Assessment software tools can generate different results for the same product system? A cause-effect analysis of the problem. Sustainable Production and Consumption, v. 20, p. 304-315, 1 out. 2019.

LUNDBLAD, L.; DAVIES, I. A. The values and motivations behind sustainable fashion consumption. Journal of Consumer Behaviour, v. 15, n. 2, p. 149-162, 1 mar. 2016.

MCTIC.

Clima.

Disponível

em:

<https://www.mctic.gov.br/mctic/opencms/ciencia/SEPED/clima/textogeral/emissao_despacho.html>. Acesso em: 27 jul. 2019.

MILÀ, L. et al. Application of life cycle assessment to footwear. International Journal of Life Cycle Assessment, v. 3, n. 4, p. 203-208, 1998.

NATTRASS, B., ALTOMARE, M. M. Ikea:" Nothing Is Impossible". Journal of Business Administration and Policy Analysis, p. 429, 1999.

OZDAMAR ERTEKIN, Z., ATIK, D. Sustainable markets: Motivating factors, barriers, and remedies for mobilization of slow fashion. Journal of Macromarketing, v. 35, n. 1, p. 53-69, 19 mar. 2015.

SHEN, B. et al. The impact of ethical fashion on consumer purchase behavior. Journal of Fashion Marketing and Management: An International Journal, v. 16, n. 2, p. 234-245, 4 maio 2012.

SHEN, B. Sustainable Fashion Supply Chain: Lessons from H\&M. Sustainability, v. 6, n. 9, p. 62366249, 2014.

STAIKOS, J. T.; RAHIMIFARD, S. Post-consumer waste management issues in the footwear 
industry. Proceedings of the Institution of Mechanical Engineers, Part B: Journal of Engineering Manufacture, v. 221, n. 2, p. 363-368, 2007.

STAIKOS, T. et al. End-of-life management of shoes and the role of biodegradable materials. End-ofLife Management of Shoes and the Role of Biodegradable Materials Theodoros, p. 497-502, 2006.

STAIKOS, T.; RAHIMIFARD, S. An End-of-Life Decision Support Tool for Product Recovery Considerations in the Footwear Industry. International Journal of Computer Integrated Manufacturing, v. 20, n. 6, p. 606-615, 2007.

TOBERGTE, D. R.; CURTIS, S. O Impacto da Reconfiguração Internacional do Mercado Calçadista sobre o Segmento Brasileiro de Couro e Calçados. Journal of Chemical Information and Modeling, v. 53, n. 9, p. 1689-1699, 2015.

TODESCHINI, B. V. et al. Innovative and sustainable business models in the fashion industry: Entrepreneurial drivers, opportunities, and challenges. Business Horizons, v. 60, n. 6, p. 759-770, 2017.

TODESCHINI, Bruna Villa; CORTIMIGLIA, Marcelo Nogueira; DE MEDEIROS, Janine Fleith. Collaboration practices in the fashion industry: Environmentally sustainable innovations in the value chain. Environmental Science \& Policy, v. 106, p. 1-11, 2020.

WIEDMANN, T.; MINX, J. A Definition of Carbon Footprint. Science, v. 1, n. 01, p. 1-11, 2007.

WINKLER, H. Closed-loop production systems-A sustainable supply chain approach. CIRP Journal of Manufacturing Science and Technology, v. 4, n. 3, p. 243-246, 2011. 